\title{
Optimal method for quantitative detection of plasma $E G F R$ T790M mutation using droplet digital PCR system
}

\author{
KEN SUZAWA $^{1}$, HIROMASA YAMAMOTO ${ }^{1}$, KADOAKI OHASHI $^{2}$, SHINSUKE HASHIDA $^{1,3}$, \\ SHUTA TOMIDA $^{4}$, TOSHIO KUBO ${ }^{2,5}$, YUHO MAKI ${ }^{1}$, JUNICHI SOH $^{1,4}$, KAZUNORI TSUKUDA $^{1}$, \\ KATSUYUKI KIURA $^{2}$, SHINICHIRO MIYOSHI ${ }^{1}$ and SHINICHI TOYOOKA ${ }^{1,3,4}$
}

\begin{abstract}
Departments of ${ }^{1}$ Thoracic Surgery, ${ }^{2}$ Hematology, Oncology and Respiratory Medicine, ${ }^{3}$ Clinical Genomic Medicine,
${ }^{4}$ Biobank, Okayama University Graduate School of Medicine, Dentistry and Pharmaceutical Sciences;

${ }^{5}$ Center for Clinical Oncology, Okayama University Hospital, Okayama, Japan
\end{abstract}

Received October 30, 2016; Accepted April 5, 2017

DOI: $10.3892 /$ or.2017.5567

\begin{abstract}
Though patients with EGFR mutations are initially responsive to EGFR-tyrosine kinase inhibitors (TKIs), most tumors ultimately acquire resistance to EGFR-TKIs. The most frequently reported mechanism is EGFR T790M mutation. In this study, using a droplet digital PCR (ddPCR) system, we assessed optimal conditions for a mutation detection assay for EGFR T790M obtained from circulating cell-free DNA (cfDNA) in plasma. The advantages of locked nucleic acids (LNA) probe, short amplicon size, and blocking oligo using peptide nucleic acids (PNA) were assessed using control DNAs from cell lines to improve the sensitivity of mutation detection. T790M alleles were then analyzed using ddPCR in 59 plasma samples from 24 NSCLC patients with EGFR mutations, and compared to the T790M status which were determined thorough re-biopsies. The assessment of the optimal assay method revealed that the assay using the short amplicon can efficiently detect more fragmented-DNA. The LNA probe and PNA clamp contributed better separation between positive and negative droplets. This PNA-LNA-ddPCR clamp method can detect mutant alleles in the sample with a mutant allele content of $0.01 \%$. In clinical plasma samples, T790M alleles were detected via ddPCR with a sensitivity of $42.8 \%$ and specificity of $97.3 \%$. We established a highly-sensitive detection assay for the T790M allele using the PNA-LNA-ddPCR clamp method.
\end{abstract}

Correspondence to: Dr Shinichi Toyooka, Department of Clinical Genomic Medicine, Okayama University Graduate School of Medicine, Dentistry and Pharmaceutical Sciences, 2-5-1 Shikata-cho, Kita-ku, Okayama 700-8558, Japan

E-mail: toyooka@md.okayama-u.ac.jp

Abbreviations: EGFR, epidermal growth factor receptor; ddPCR, droplet digital PCR; LNA, locked nucleic acids; PNA, peptide nucleic acids

Key words: T790M mutation, digital PCR, circulating DNA, locked nucleic acid
ddPCR is a promising method for detecting non-invasive T790M mutation.

\section{Introduction}

Recently, various cancer driver gene mutations have been identified in non-small cell lung cancers (NSCLC), including epidermal growth factor receptor (EGFR), KRAS, HER2, and $B R A F$ mutations (1). Personalized treatment based on this molecular genetic information showed positive therapeutic effects (2). EGFR mutations, mostly exon 19 deletions and L858R point mutation in exon 21 , are associated with clinical benefits from EGFR tyrosine kinase inhibitors (EGFR-TKIs) (3). Therefore, somatic mutational analysis has become a routine part of clinical practice and is indispensable for the treatment decisions in NSCLC clinical settings. Though patients with EGFR mutations are initially responsive to EGFR-TKIs, almost all of their tumors ultimately acquire TKI resistance as a result of tumor heterogeneity or clonal evolution $(4,5)$. Various mechanisms of resistance to the first generation EGFR-TKIs, including gefitinib and erlotinib, have been identified, and the drugs to overcome resistance have been developed. For example, the most frequently reported mechanism of acquired resistance to EGFR-TKIs is a EGFR T790M point mutation in exon 20 , consisting of $>50 \%$ of these cases $(4,6)$. Third-generation EGFR TKIs, including WZ4002, CO-1686, and AZD9291, have been developed and potently appear to inhibit tumors with EGFR activating mutations even in the presence of the T790M mutation (7). Thus, the importance of T790M mutation detection is increasing in NSCLC treatment. However, the conventional method for mutation detection requires a cancer cell biopsy, which is an invasive procedure and sometimes difficult to perform repeatedly.

Circulating cell-free DNA (cfDNA) has been shown to be released from apoptotic and necrotic cells into the circulation and has been identified in plasma (8). Recent technological development has demonstrated the potential of molecular genetic profiling from cfDNA. cfDNA has received great attention as one of the critical targets for non-invasive mutation detection (9). It enables repetitive biopsy and monitoring of cancer status, though detection of cfDNA from tumor cells 
in the circulation still remains challenging. The detection of cfDNA from tumor cells requires a highly-sensitive assay, because the majority of circulating cfDNA appears to be from normal tissue (10). Droplet digital PCR (ddPCR) is one of the digital PCR technologies and provides highly-sensitive and absolute quantitative detection of target genes (9). In ddPCR, template DNA is partitioned into approximately 20,000 droplets in a single reaction well, and then amplified within individual droplets. This approach facilitates the detection and quantification of rare mutants in a background of wild-type alleles, because rare mutant alleles can avoid competition with wild-type ones. However, the optimal method for detecting cfDNA remains debatable.

In this study, we evaluated the optimal method for mutation analysis with EGFR T790M mutation in plasma using a ddPCR system.

\section{Materials and methods}

Clinical samples and cell lines. This study was approved by the ethics committee of Okayama University (receipt number: 2220). All patients enrolled in the study provided written informed consent for the use of tissue and blood sample. July 2014 through February 2015, we obtained 7-ml peripheral blood samples using EDTA-2K tubes from two healthy volunteers and 24 lung adenocarcinoma patients with EGFR mutations. These patients had been treated with EGFR-TKI and their EGFR T790M mutational status was examined through re-biopsy as a part of clinical care. Clinical EGFR mutation tests in tissue samples had been performed before entry into the study by an commercial clinical laboratory via mutant-enriched PCR assay using the peptide nucleic acid (PNA)-locked nucleic acids (LNA) PCR clamp method (LSI Medience Corp., Tokyo, Japan) $(11,12)$. The approval of the Institutional Review Board and informed consent from each patient were obtained. After drawing blood, samples were immediately centrifuged at $2,330 \mathrm{x}$ g for $10 \mathrm{~min}$ to collect plasma, and the plasma samples were centrifuged at $16,000 \mathrm{x} \mathrm{g}$ for 10 additional $\mathrm{min}$. The supernatants were collected, frozen, and stored at $-80^{\circ} \mathrm{C}$. In addition, we used an immortalized normal human bronchial epithelial cell line (HBEC-5KT) harboring the wild-type EGFR gene and the NCI-H1975 cell line (H1975) harboring the EGFR mutations L858R and T790M as negative and positive controls, respectively $(13,14)$. HBEC-5KT and H1975 were kindly provided by Dr Adi F. Gazdar (The University of Texas Southwestern Medical Center, Dallas, TX, USA).

DNA extraction, quantification, and fragmentation. After thawing the frozen plasma samples, DNA was extracted using QIAamp Circulating Nucleic Acid kit (Qiagen, Venlo, The Netherlands), and eluted in $20 \mu \mathrm{l}$ of the kit's elution buffer. Control DNA from cell lines was extracted using DNeasy Blood and Tissue kit (Qiagen) and fragmented through sonication to obtain an average size of $200 \mathrm{bp}$ using Covaris M220 Focused-ultrasonicator (Covaris Inc., Woburn, MA, USA) to closely mimic cfDNA in plasma $(15,16)$. DNA size distribution was analyzed using Agilent 2200 TapeStation (Agilent Technologies, Santa Clara, CA, USA). Genomic DNA was quantified by Qubit 2.0 Fluorometer and Qubit
dsDNA HS or BR assay kit (Thermo Fisher Scientific, Inc., Waltham, MA, USA).

Primers and probes. The sequences of primers, probes, and blocking oligo used in this study are shown in Table I. S1 and AS1 primers and the M1 probe, which were designed in a previous study, were used as a validated reference assay (9). The primer melting temperatures $(\mathrm{Tm})$ were calculated using Primer3Plus (http://primer3plus.com/). Probes, including LNA, were designed using IDT Biophysics software (http:// biophysics.idtdna.com/) and synthesized by IDT-MBL KK (Nagoya, Japan). The blocking oligo using PNA was synthesized by Fasmac Co., Ltd. (Atsugi, Japan).

Droplet digital PCR assay for EGFR T790M detection. ddPCR was performed using QX200 Droplet Digital PCR system (Bio-Rad, Hercules, CA, USA). The final volume of the PCR mixture was $20 \mu \mathrm{l}$, containing $10 \mu \mathrm{l}$ of ddPCR Supermix for Probes (No dUTP) (Bio-Rad), $1 \mu \mathrm{M}$ of each primer, $0.25 \mu \mathrm{M}$ of each probe, $200 \mu \mathrm{M}$ of dNTP, and $5 \mu \mathrm{l}$ of DNA (from approximately $2 \mathrm{ml}$ of blood sample or control DNA), with or without $5 \mu \mathrm{M}$ of blocking oligo. The following PCR conditions for ddPCR were used: 1) an initial denaturation step at $95^{\circ} \mathrm{C}$ for $10 \mathrm{~min}$ followed by 2) 45 cycles at $94^{\circ} \mathrm{C}$ for $30 \mathrm{sec}$, and 3) 45 cycles at $57^{\circ} \mathrm{C}$ for $1 \mathrm{~min}$. Each ramp rate was $2^{\circ} \mathrm{C}$ per second. Annealing temperatures were optimized by gradient PCR (data not shown). PCR products were then subjected to analysis by the QX-200 droplet reader and QuantaSoft analysis software (Bio-Rad). Assays were considered positive if $>3$ droplets were over the threshold fluorescence of 8,000 . The fluorescence threshold and cut off number for mutation detection were determined by assessing these data using positive and negative control DNA from cell lines.

Statistical analysis. The concentrations of target alleles were calculated using QuantaSoft software 9.2.1 (Bio-Rad) based on Poisson distribution.

\section{Results}

The size of circulating cell-free DNA. The size distribution of cfDNA extracted from healthy donors and lung cancer patients was assessed (Fig. 1A). It showed similar distribution patterns between healthy donors and lung cancer patients, and two peaks were identified in both sets of patients. The first peak was distributed from 128 to 168 bp (median, $158 \mathrm{bp}$ ), and the 2nd peak was distributed from 526 to $797 \mathrm{bp}$ (median: $681 \mathrm{bp}$ ). Next, we prepared control cfDNA mimic samples through sonication to evaluate the method of highlysensitive detection with focus on cfDNA; the size of the samples was then confirmed. After sonication, the genomic DNA samples were fragmented to an average size of approximately 200 bp (Fig. 1B).

The effect of LNA probe to suppress the fluorescence intensity of negative droplets. To examine the advantage of using the LNA probe, ddPCR was performed using different probes consisting of a regular oligonucleotide probe (M1) and an LNA probe (M2) and the same primers (S1 and AS1). These probes were designed to display similar Tms, and were based either 
Table I. Sequences of primers and probes for ddPCR.

\begin{tabular}{lllcc}
\hline Oligo name & \multicolumn{1}{c}{ Oligo sequenses 5' to 3' } & $\begin{array}{c}\text { Tm } \\
\left({ }^{\circ} \mathrm{C}\right)\end{array}$ & $\begin{array}{c}\text { Product } \\
\text { size (bp) }\end{array}$ & $\begin{array}{c}\text { Match-mismatch } \\
\text { Tm difference }\left({ }^{\circ} \mathrm{C}\right)\end{array}$ \\
\hline Primer S1 & GCCTGCTGGGCATCTG & 59.5 & 93 & NA \\
Primer AS1 & TCTTTGTGTTCCCGGACATAGTC & 61.6 & & NA \\
Primer S2 & CCTCACCTCCACCGTG & 57.8 & 43 & NA \\
Primer AS2 & CGAAGGGCATGAGCTG & 56.6 & & NA \\
Probe M1 & FAM/ATGAGCTGCATGATGAG/IABkFQ & 58.83 & NA & 5.57 \\
Probe M2 & FAM/TCA+TC+A+T+GC+AGC/IABkFQ & 60.7 & NA & 11.41 \\
Blocking oligo & TCATCACGCAG (Peptide nucleic acids) & 58.6 & NA & NA
\end{tabular}

ddPCR, droplet digital PCR; Tm, melting temperature; IABkFQ, Iowa Black FQ; NA, not available. Locked nucleic acid: +N.

A

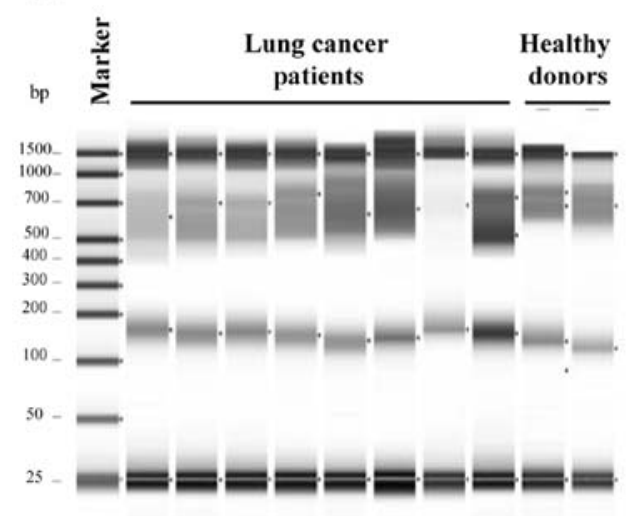

Lung cancer patient
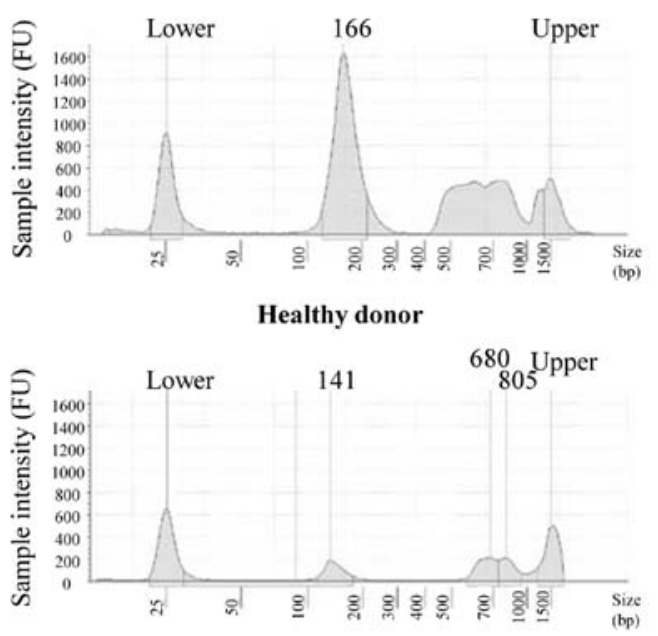

B
HBEC-5KT

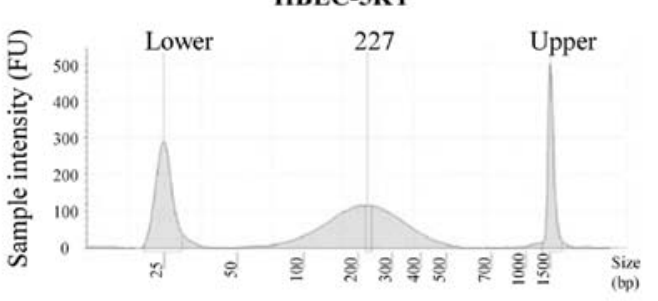

H1975

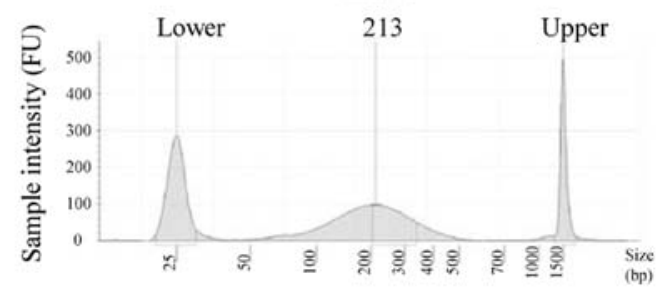

Figure 1. Comparison of electropherogram of cfDNA samples. (A) The size distribution of cfDNA in plasma extracted from lung cancer patients and healthy donors were analyzed using Agilent 2200 TapeStation. Normalization was performed using two internal markers, visible as high, narrow amplitudes at 25 and $1,500 \mathrm{bp}$, respectively. (B) The genomic DNAs from cell lines, HBEC-5KT and H1975, were extracted and fragmented to an average size of approximately 200 bp by sonication. DNA size distribution was then subjected to be analyzed using Agilent 2200 TapeStation.

on regular oligonucleotides or LNA. The match-mismatch Tm difference of the regular oligonucleotides and LNA probes were $5.6^{\circ} \mathrm{C}$ and $11.4^{\circ} \mathrm{C}$, respectively. As shown in Fig. $2 \mathrm{~A}$, the fluorescence intensity of negative droplets was suppressed in the LNA 
A

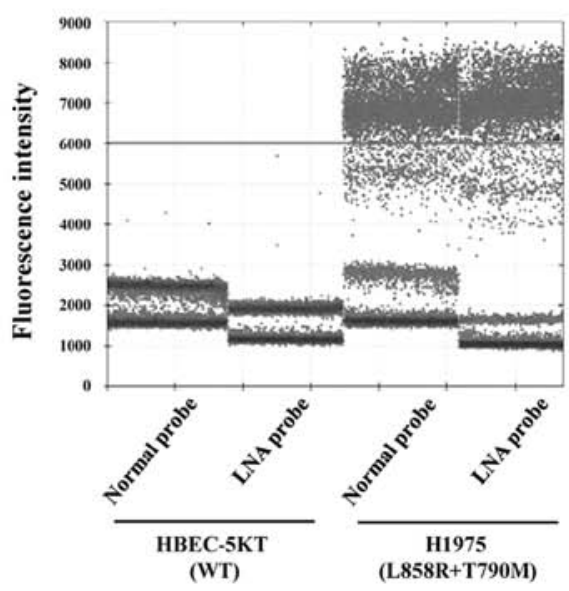

B

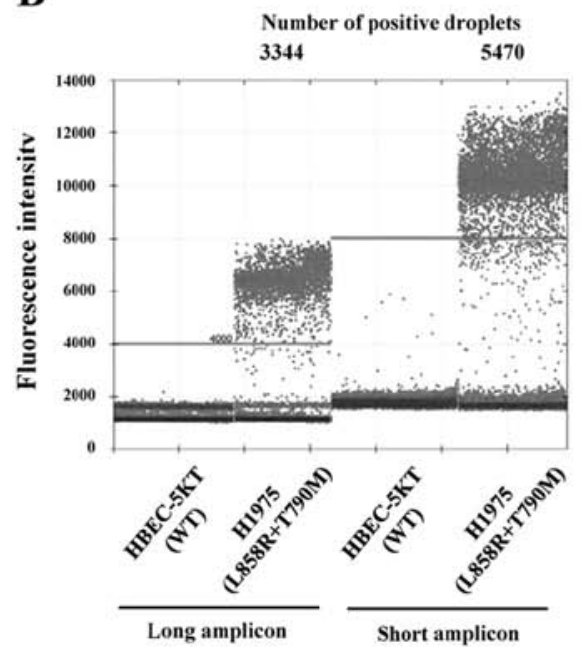

C
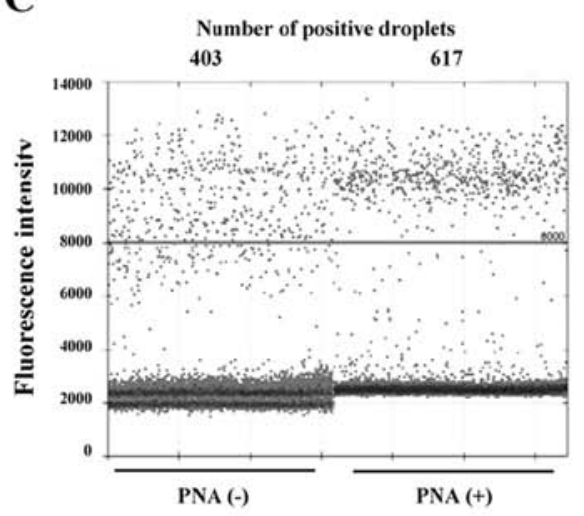

Figure 2. The optimization of mutation analysis in plasma samples using droplet digital PCR (ddPCR) system. (A) Control DNA of HBEC-5KT cells harboring the wild-type EGFR and H1975 cells harboring the EGFR mutations L858R and T790M was analyzed by ddPCR assay using regular oligonucleotide probe versus LNA probe. The fluorescence intensity of each droplet is shown. (B) The fragmented template DNA of HBEC-5KT and H1975 was analyzed by ddPCR assay using either of two primer sets, of which amplicon size was 43 bp versus $92 \mathrm{bp}$. Threshold fluorescence was set at 4,000 for long amplicon and 8,000 for short amplicon, respectively, and the number of positive droplets were counted. (C) Diluted DNA mixture (100 ng) with a mutant content of only $1 \%$ were analyzed with or without the PNA clamp. Threshold fluorescence was set at 8,000, and the number of positive droplets were counted.

probe group compared with the regular oligonucleotide probe group in HBEC-5KT cells as negative controls. In H1975 cells, the negative droplet suppression by the LNA probe contributed to better separation between both positive and negative droplet clusters. These results suggest that the LNA probe displayed an improvement in mismatch discrimination, which was thought to possibly result in better specificity.

Improvement in the detection of cfDNA with short-size amplicon. Next, we assessed the amplicon size for better detection of cfDNA. Since cfDNA is highly fragmented, we hypothesized that ddPCR with a short-size amplicon improves the detection efficiency of cfDNA. We designed new primers (S2 and AS2), the amplicon size of which was $43 \mathrm{bp}$, whereas the amplicon size of the validated reference primers ( $\mathrm{S} 1$ and AS1) was $93 \mathrm{bp}$. ddPCR was performed using either of the two primer sets and the same LNA probe (M2). The template DNA from either HBEC-5KT or H1975 was fragmented through sonication to approximately $200 \mathrm{bp}$ as previously mentioned. The use of a short-size amplicon led to approximately a 1.6-fold increase in the number of detected positive droplets (Fig. 2B). On the other hand, there were no significant differences in the number of false positive droplets between the two primer sets. This approach with short-size amplicon may be useful to increase cfDNA detection sensitivity because cfDNA is highly fragmented.

Enrichment of the PCR efficiency of targeted mutation alleles with PNA clamping method. Since plasma cfDNA contains abundant cfDNA released from normal cells, a highly-sensitive method which enables mutations to be selectively enriched in a large background of wild-type alleles is preferable. We prepared a blocking oligo using PNA to suppress PCR amplification of the wild-type alleles and $100 \mathrm{ng}$ diluted DNA mixture with a mutant content of only $1 \%$ was analyzed with or without the PNA clamp. The median positive droplet fluorescence intensity

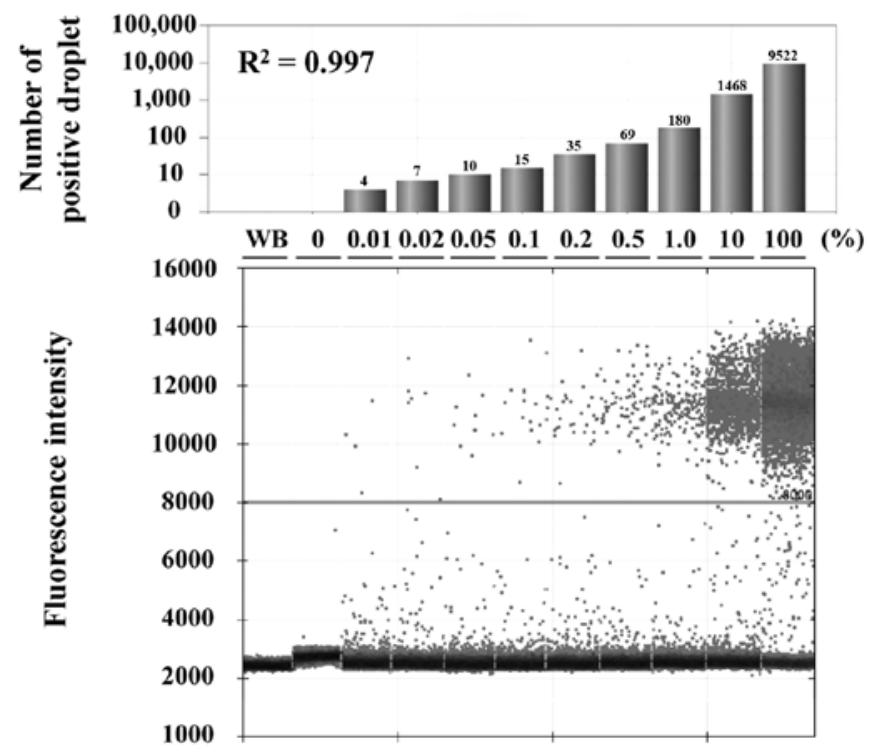

Figure 3. Detection limit and quantitative ability of PNA-LNA-ddPCR clamp method for EGFR T790M detection. Total $50 \mathrm{ng}$ of serially diluted DNA mixtures of H1975 and HBEC-5KT was applied and droplet digital PCR (ddPCR) was performed using LNA probe (M2), short-size amplicon primers (S2 and AS2), and PNA clamp. WB, water blank.

was increased in the group with the PNA clamp compared to the one without the clamp, which resulted in better separation between positive and negative droplets clusters. The use of the PNA clamp led to approximately a 1.5 -fold increase in the number of detected positive droplets (Fig. 2C). This approach appeared to enhance PCR efficiency for T790M alleles by blocking the amplification of wild-type alleles.

Detection limit and quantitative ability of ddPCR for EGFR T790M detection. The detection limit and quantitative ability of this assay were determined using serially diluted DNA 
Table II. Patient characteristics $(n=24)$.

\begin{tabular}{lr}
\hline Subsets & $\mathrm{n}(\%)$ \\
\hline Median age, (range), year & $67(39-84)$ \\
Sex & \\
Male & $7(29.2 \%)$ \\
Female & $17(70.8 \%)$ \\
Smoking history & \\
Smoked & $9(37.5 \%)$ \\
Never-smoked & $15(62.5 \%)$ \\
Drug-sensitive EGFR mutation & $7(29.2 \%)$ \\
L858R & $16(66.7 \%)$ \\
Exon 19 deletion & $1(4.2 \%)$ \\
G719A & \\
Tissue T790M mutation & \\
(PNA-LNA Clamp method) & $9(37.5 \%)$ \\
Positive & $15(62.5 \%)$ \\
Negative & \\
\hline
\end{tabular}

Table III. Correlation of EGFR T790M mutational status between cfDNA and rebiopsy tissue DNA.

\begin{tabular}{lccc}
\hline & \multicolumn{2}{c}{$\begin{array}{c}\text { EGFR T790M status } \\
\text { in rebiopsy tissue-DNA } \\
\text { (PNA-LNA PCR clamp method) }\end{array}$} & \\
\cline { 2 - 3 } & Positive & Negative & Sum \\
\hline $\begin{array}{l}\text { EGFR T790M status } \\
\text { in cfDNA (ddPCR) }\end{array}$ & & 1 & \\
Positive & 9 & 37 & 10 \\
Negative & 12 & 38 & 59 \\
Sum & 21 & 38 \\
\hline
\end{tabular}

PNA, peptide nucleic acid; LNA, locked nucleic acid.

mixtures of H1975 and HBEC-5KT. In total, $50 \mathrm{ng}$ of template DNA was used for this assay, and ddPCR was performed using LNA probe (M2), short amplicon primers (S2 and AS2), and a PNA clamp. Results indicated that the PNA-LNA-ddPCR clamp method can detect mutant alleles in the sample with a mutant content of $0.01 \%$, and the concentration of detectedmutant alleles correlated with the applied control DNA mixture concentrations in a linear fashion $\left(\mathrm{R}^{2}=0.997\right)($ Fig. 3).

EGFR T790M detection and monitoring in clinical plasma samples. We examined T790M mutation by ddPCR using the PNA-LNA-ddPCR clamp method for clinical plasma samples. The patient characteristics are shown in Table II. All patients had EGFR-mutant NSCLC and had received treatment with an EGFR-TKI, and 9 patients were positive for T790M in tumor re-biopsy. Of the 59 clinical plasma samples, T790M alleles were detected via ddPCR in 10 samples. As 21 of 59 plasma
A
Pre-3rd generation EGFR-TKI

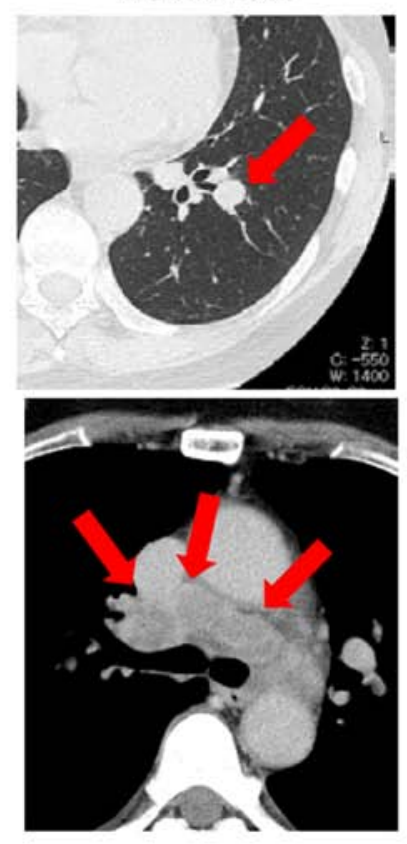

B

\section{Post-3rd generation} EGFR-TKI
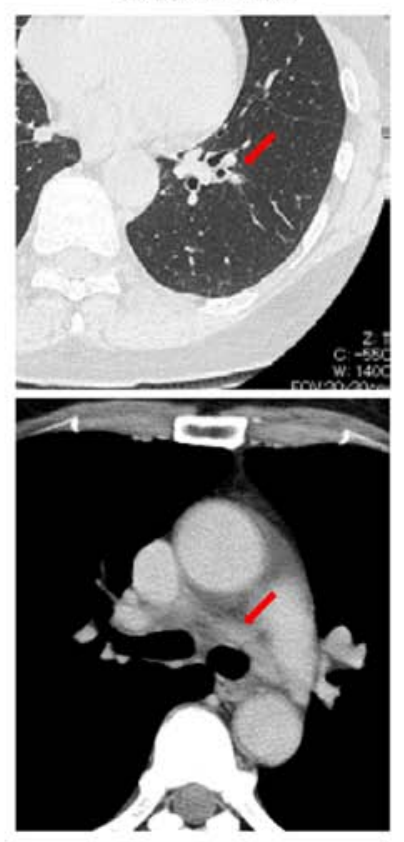

Treatment by 3rd-generation EGFR-TKI

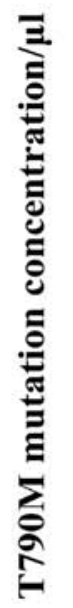

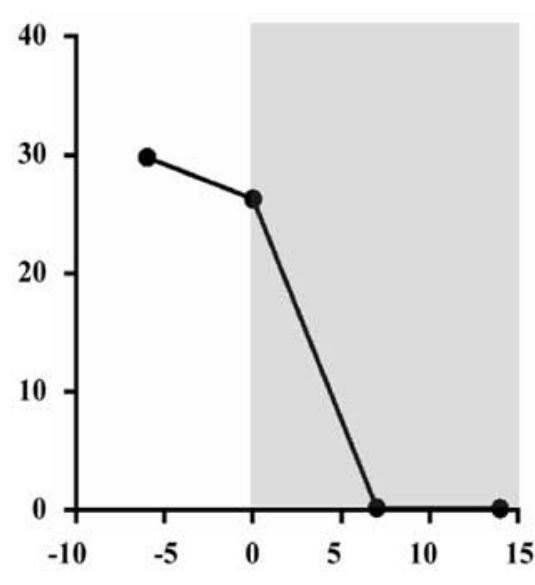

Days on treatment

Figure 4. EGFR T790M monitoring in clinical plasma samples. (A) Contrastenhanced CT images of disease in the left lower lobe of the lung and mediastinal lymph nodes (arrows) of pre-treatment (left) and after 6 weeks of 3rd-generation EGFR-TKI treatment (right). (B) Serial cfDNA T790M mutant alleles in plasma was dynamically monitored by PNA-LNA-ddPCR clamp method.

samples were collected from 9 patients with T790M positive in re-biopsy sample, the sensitivity of ddPCR was $42.8 \%$. Among 10 positive samples in the ddPCR assay, nine were concordant with the T790M mutational status of the re-biopsy samples, and one was disconcordant. ddPCR specificity was determined as $97.3 \%$. Results are summarized in Table III. In patients diagnosed as T790M-positive in plasma, one patient was enrolled in this study before treatment with thirdgeneration EGFR-TKI after acquiring gefitinib resistance. We compared cfDNA T790M mutant alleles in pre- versus post-treatment plasma (Fig. 4). It revealed that the number of 
T790M alleles from plasma cfDNA rapidly decreased after third-generation EGFR-TKI treatment. This decline in plasma cfDNA was consistent with the computed tomography (CT) images. Six weeks after the administration of third-generation EGFR-TKI treatment, contrast-enhanced CT examination revealed that the primary tumor in the left lower lobe of the lung and mediastinal lymph nodes had significantly shrunk.

\section{Discussion}

The emergence of a non-invasive mutational analysis using cfDNA enables both repetitive disease assessment and monitoring. However, mutation detection still remains challenging because circulating bloodstream cfDNA derived from tumor tissue is highly fragmented, yields small quantities, and presents at very low allelic frequencies in abundant cfDNA released from normal cells in nature $(10,17)$. To overcome these difficulties, in this study, we assessed the optimal approach using ddPCR for mutation detection from plasma cfDNA.

In this study, the median cfDNA concentration from lung cancer patients was $1.4 \mathrm{ng} / \mu \mathrm{l}$, and the total amount in single $7 \mathrm{ml}$ peripheral blood samples was only approximately $28 \mathrm{ng}$, which equates to 8,400 copies of human haploid genomic equivalents. Therefore, without consideration of copy number gain of mutant alleles, the theoretical mutation detection limit for cfDNA in one blood sample shows a sensitivity of $0.012 \%$, indicating one mutant allele in a background of 8,400 wild-type alleles.

Moreover, amplicon size is also a principal issue for mutation analysis of cfDNA in plasma samples, because cfDNA has been shown to be highly fragmented after nucleosomal cleavage in apoptosis (17). As for cell-free fetal DNA, Sikora et al reported that a real-time PCR assay with the 50 bp amplicon was able to detect 1.6 -fold more cell-free fetal DNA than the conventional real-time PCR assay with a longer amplicon (18). We confirmed that the assay with the $42 \mathrm{bp}$ amplicon was able to detect 1.6-fold more fragmented template DNA when compared with the assay for the $93 \mathrm{bp}$ amplicon. Our results in this study were consistent with previous results. This approach may be useful for all assays in which target DNA is fragmented, including DNA from formalin-fixed paraffin-embedded materials.

One of the advantages of digital PCR is the partitioning of competing backgrounds of wild-type alleles, leading to decrease in their PCR inhibitory effects and improvements in detection sensitivity (19). However, when DNA mixtures of positive and negative controls were subjected to ddPCR assay, some components, possibly false negatives, distributed between positive and negative clusters. The fluorescent signal was higher than that of negative clusters, but showed a weak signal when compared with positive clusters. We hypothesized that one of the reasons for these false negative droplets was PCR inhibitory effects because of a pool of wild-type alleles. PNA is resistant to the 5' nuclease activity of Taq DNA polymerase, leading to a PNA oligo with a superior clamp primer for inhibiting PCR amplification of wild-type sequences. Nagai et al reported that an EGFR mutation detection system using PNA-clamping PCR with a melting curve analysis can detect $E G F R$ mutations in the presence of 100- to 1,000-fold background of wild-type EGFR (11). In this study, we showed that the PNA clamping method in ddPCR also enriched the PCR efficiency of targeted mutation alleles. These strategies resulted in highly-sensitive ddPCR assay that enables detection of one mutant allele in the presence of 10,000 background wildtype alleles. A potential disadvantage of the PNA clamping PCR is that the mutant frequency cannot be measured because of the suppression of a wild-type allele control by PNA clamping. However, ddPCR can provide absolute quantitation of target sequences in the loaded DNA sample, and the levels of plasma DNA concentrations in cancer patients is known to be associated with tumor burden and malignant progression (15). Therefore, the number of mutant fragments in the loaded DNA sample are also deemed to represent the tumor biology, same as mutant frequency.

Of the 59 clinical plasma samples, T790M alleles in 10 samples were successfully detected by ddPCR. The sensitivity of ddPCR was $42.8 \%$, and the specificity was $97.3 \%$ when referred to that of diagnostic tissue biopsies determined by clinical routine analysis using the PNA-LNA PCR clamp method. In 12 plasma samples, our assay could not identify the T790M mutation that had been detected in the corresponding biopsy samples, and in one plasma sample, the T790M mutation was identified only in the plasma sample. However, the limitation of this study can be identified. The plasma samples were obtained at different times from the tissue re-biopsy tests. The median time interval between plasma collection and re-biopsy test was 12.5 months. Therefore, we have to consider these different timings of sample collections and heterogeneity of tumor cells, and the discrepancy between the two results could be because of biology and not technological capability. Every mutation may not necessarily be represented in a patient's circulation system. Furthermore, the amount of template DNA is also a critical factor for detection of rare mutations from blood samples. Even though the assay can achieve a better sensitivity of $0.01 \%$, one in 10,000 alleles, it is impossible to maximize its ability without applying $>10,000$ alleles. However, in practice, it is often difficult to apply 10,000 alleles because of the limitation of blood sampling volume. Recently, a $B R A F$ mutation detection system using cfDNA from urine samples was reported $(20,21)$. In these studies, 60-120 $\mathrm{ml}$ of urine was collected for a urinary cfDNA assay. Urine sampling is less invasive and more suitable for repeated and large volume sampling than blood, which may solve the problem with small template DNA amounts.

In conclusion, we showed that the use of a short-size amplicon, LNA probe, and PNA blocker improve the sensitivity of a mutation detection for cfDNA samples. ddPCR is a promising method for non-invasive T790M mutation detection.

\section{Acknowledgements}

The authors thank Dr Mizuki Morita (Biorepository Research and Networking, Okayama University Graduate School of Medicine, Dentistry and Pharmaceutical Sciences, Okayama, Japan), Ms. Fumiko Isobe (Department of Thoracic, Breast and Endocrinological Surgery, Okayama University Graduate School of Medicine, Dentistry and Pharmaceutical Sciences, Okayama, Japan), Dr Takehiro Matsubara, Ms. Yuko Hanafusa and Ms. Yayoi Kubota (Biobank of Okayama University Hospital, Okayama, Japan) for their technical support. 


\section{References}

1. Collisson EA, Campbell JD, Brooks AN, Berger AH, Lee W, Chmielecki J, Beer DG, Cope L, Creighton CJ, Danilova L, et al; Cancer Genome Atlas Research Network: Comprehensive molecular profiling of lung adenocarcinoma. Nature 511: 543-550, 2014

2. Kris MG, Johnson BE, Berry LD, Kwiatkowski DJ, Iafrate AJ, Wistuba II, Varella-Garcia M, Franklin WA, Aronson SL, Su PF, et al: Using multiplexed assays of oncogenic drivers in lung cancers to select targeted drugs. JAMA 311: 1998-2006, 2014.

3. Mok TS, Wu YL, Thongprasert S, Yang CH, Chu DT, Saijo N, Sunpaweravong P, Han B, Margono B, Ichinose Y, et al: Gefitinib or carboplatin-paclitaxel in pulmonary adenocarcinoma. N Engl J Med 361: 947-957, 2009.

4. Sequist LV, Waltman BA, Dias-Santagata D, Digumarthy S, Turke AB, Fidias P, Bergethon K, Shaw AT, Gettinger S, Cosper AK, et al: Genotypic and histological evolution of lung cancers acquiring resistance to EGFR inhibitors. Sci Transl Med 3: $75 \mathrm{ra} 26,2011$.

5. Soucheray M, Capelletti M, Pulido I, Kuang Y, Paweletz CP, Becker JH, Kikuchi E, Xu C, Patel TB, Al-Shahrour F, et al: Intratumoral heterogeneity in EGFR-Mutant NSCLC results in divergent resistance mechanisms in response to EGFR tyrosine kinase inhibition. Cancer Res 75: 4372-4383, 2015.

6. Kobayashi S, Boggon TJ, Dayaram T, Jänne PA, Kocher O, Meyerson M, Johnson BE, Eck MJ, Tenen DG and Halmos B: EGFR mutation and resistance of non-small-cell lung cancer to gefitinib. N Engl J Med 352: 786-792, 2005.

7. Cross DA, Ashton SE, Ghiorghiu S, Eberlein C, Nebhan CA, Spitzler PJ, Orme JP, Finlay MR, Ward RA, Mellor MJ, et al: AZD9291, an irreversible EGFR TKI, overcomes T790Mmediated resistance to EGFR inhibitors in lung cancer. Cancer Discov 4: 1046-1061, 2014.

8. Crowley E, Di Nicolantonio F, Loupakis F and Bardelli A: Liquid biopsy: Monitoring cancer-genetics in the blood. Nat Rev Clin Oncol 10: 472-484, 2013.

9. Oxnard GR, Paweletz CP, Kuang Y, Mach SL, O'Connell A, Messineo MM, Luke JJ, Butaney M, Kirschmeier P, Jackman DM, et al: Noninvasive detection of response and resistance in EGFRmutant lung cancer using quantitative next-generation genotyping of cell-free plasma DNA. Clin Cancer Res 20: 1698-1705, 2014.

10. Diaz LA Jr and Bardelli A: Liquid biopsies: Genotyping circulating tumor DNA. J Clin Oncol 32: 579-586, 2014.

11. Nagai Y, Miyazawa H, Huqun, Tanaka T, Udagawa K, Kato M, Fukuyama S, Yokote A, Kobayashi K, Kanazawa M, et al: Genetic heterogeneity of the epidermal growth factor receptor in non-small cell lung cancer cell lines revealed by a rapid and sensitive detection system, the peptide nucleic acid-locked nucleic acid PCR clamp. Cancer Res 65: 7276-7282, 2005.
12. Tanaka T, Nagai Y, Miyazawa H, Koyama N, Matsuoka S, Sutani A, Huqun, Udagawa K, Murayama Y, Nagata M, et al: Reliability of the peptide nucleic acid-locked nucleic acid polymerase chain reaction clamp-based test for epidermal growth factor receptor mutations integrated into the clinical practice for non-small cell lung cancers. Cancer Sci 98: 246-252, 2007.

13. Ramirez RD, Sheridan S, Girard L, Sato M, Kim Y, Pollack J, Peyton M, Zou Y, Kurie JM, Dimaio JM, et al: Immortalization of human bronchial epithelial cells in the absence of viral oncoproteins. Cancer Res 64: 9027-9034, 2004.

14. Gandhi J, Zhang J, Xie Y, Soh J, Shigematsu H, Zhang W, Yamamoto H, Peyton M, Girard L, Lockwood WW, et al: Alterations in genes of the EGFR signaling pathway and their relationship to EGFR tyrosine kinase inhibitor sensitivity in lung cancer cell lines. PLoS One 4: e4576, 2009.

15. Schwarzenbach H, Hoon DS and Pantel K: Cell-free nucleic acids as biomarkers in cancer patients. Nat Rev Cancer 11: 426-437, 2011.

16. Giacona MB, Ruben GC, Iczkowski KA, Roos TB, Porter DM and Sorenson GD: Cell-free DNA in human blood plasma: Length measurements in patients with pancreatic cancer and healthy controls. Pancreas 17: 89-97, 1998.

17. Heitzer E, Ulz P and Geigl JB: Circulating tumor DNA as a liquid biopsy for cancer. Clin Chem 61: 112-123, 2015.

18. Sikora A, Zimmermann BG, Rusterholz C, Birri D, Kolla V, Lapaire O, Hoesli I, Kiefer V, Jackson L and Hahn S: Detection of increased amounts of cell-free fetal DNA with short PCR amplicons. Clin Chem 56: 136-138, 2010.

19. Weisenberger DJ, Trinh BN, Campan M, Sharma S, Long TI, Ananthnarayan S, Liang G, Esteva FJ, Hortobagyi GN, McCormick F, et al: DNA methylation analysis by digital bisulfite genomic sequencing and digital MethyLight. Nucleic Acids Res 36: 4689-4698, 2008

20. Hyman DM, Diamond EL, Vibat CR, Hassaine L, Poole JC, Patel M, Holley VR, Cabrilo G, Lu TT, Arcila ME, et al: Prospective blinded study of BRAFV600E mutation detection in cell-free DNA of patients with systemic histiocytic disorders. Cancer Discov 5: 64-71, 2015.

21. Janku F, Vibat CR, Kosco K, Holley VR, Cabrilo G, MericBernstam F, Stepanek VM, Lin PP, Leppin L, Hassaine L, et al: BRAF V600E mutations in urine and plasma cell-free DNA from patients with Erdheim-Chester disease. Oncotarget 5: 3607-3610, 2014. 